\title{
Fusion-Fission Dynamics and Perspectives of Future Experiments*
}

\author{
V. I. Zagrebaev*** M. G. Itkis, and Yu. Ts. Oganessian \\ Flerov Laboratory of Nuclear Reactions, Joint Institute for Nuclear Research, \\ Dubna, Moscow oblast, 141980 Russia
}

Received September 2, 2002

\begin{abstract}
The paper is focused on reaction dynamics of superheavy-nucleus formation and decay at beam energies near the Coulomb barrier. The aim is to review the things we have learned from recent experiments on fusion-fission reactions leading to the formation of compound nuclei with $Z \geq 102$ and from their extensive theoretical analysis. Major attention is paid to the dynamics of formation of very heavy compound nuclei taking place in strong competition with the process of fast fission (quasifission). The choice of collective degrees of freedom playing a fundamental role and finding the multidimensional driving potential and the corresponding dynamic equation regulating the whole process are discussed. A possibility of deriving the fission barriers of superheavy nuclei directly from performed experiments is of particular interest here. In conclusion, the results of a detailed theoretical analysis of available experimental data on the "cold" and "hot" fusion-fission reactions are presented. Perspectives of future experiments are discussed along with additional theoretical studies in this field needed for deeper understanding of the fusion-fission processes of very heavy nuclear systems. (C) 2003 MAIK "Nauka/Interperiodica".
\end{abstract}

\section{INTRODUCTION}

The interest in the synthesis of superheavy nuclei has grown lately due to new experimental results [13] demonstrating a real possibility of producing and investigating the nuclei in the region of the so-called "island of stability." The new reality demands more substantial theoretical support of these expensive experiments, which will allow a more reasonable choice of fusing nuclei and collision energies as well as a better estimation of the cross sections and unambiguous identification of evaporation residues (ERs).

A whole process of superheavy-nucleus formation can be divided into three reaction stages. At the first stage, colliding nuclei overcome the Coulomb barrier and approach the point of contact $R_{\text {cont }}=R_{1}+$ $R_{2}$. Quasielastic and deep-inelastic reaction channels dominate at this stage, leading to formation of projectile-like and target-like fragments (PLF and TLF) in the exit channel. At subbarier energies, only a small part of incoming flux with low partial waves reaches the point of contact. Denote the corresponding probability as $P_{\text {cont }}(l, E)$. Experiments on deepinelastic collisions and our knowledge about nuclear friction forces allow us to conclude that, at the contact point, nuclei have almost zero kinetic energy. At the second reaction stage, touching nuclei evolve into the configuration of an almost spherical compound

\footnotetext{
* This article was submitted by the authors in English.

** e-mail: valeri.zagrebaev@jinr.ru
}

mononucleus. For light or very asymmetric nuclear systems, this evolution occurs with a probability close to unity. Two touching heavy nuclei after dynamic deformation and exchange by several nucleons may reseparate into PLF and TLF or may go directly to fission channels without formation of a compound nucleus. The later process is usually called quasifission. Denote a probability for two touching nuclei to form the compound nucleus $(\mathrm{CN})$ as $P_{\mathrm{CN}}(l, E)$. At the third reaction stage, the $\mathrm{CN}$ emits neutrons and $\gamma$ rays, lowering its excitation energy and finally forming the residual nucleus in its ground state. This process takes place in strong competition with fission (normal fission), and the corresponding survival probability $P_{x n}\left(l, E^{*}\right)$ is usually much less than unity even for a weakly excited superheavy nucleus.

Thus, the production cross section of a cold residual nucleus $B$, which is the product of neutron evaporation and $\gamma$ emission from an excited compound nucleus $C$, formed in the fusion process of two heavy nuclei $A_{1}+A_{2} \rightarrow C \rightarrow B+x n+N \gamma$ at c.m. energy $E$ close to the Coulomb barrier in the entrance channel, can be decomposed over partial waves and written as

$$
\begin{aligned}
& \sigma_{\mathrm{ER}}^{x n}(E) \approx \frac{\pi \hbar^{2}}{2 \mu E} \sum_{l=0}^{\infty}(2 l+1) P_{\mathrm{cont}}(E, l) \\
\times & P_{\mathrm{CN}}\left(A_{1}+A_{2} \rightarrow C ; E, l\right) P_{x n}\left(C \rightarrow B ; E^{*}, l\right) .
\end{aligned}
$$

Different theoretical approaches are used for analyzing all three reaction stages. However, the dynamics of the intermediate stage of the $\mathrm{CN}$ forma- 


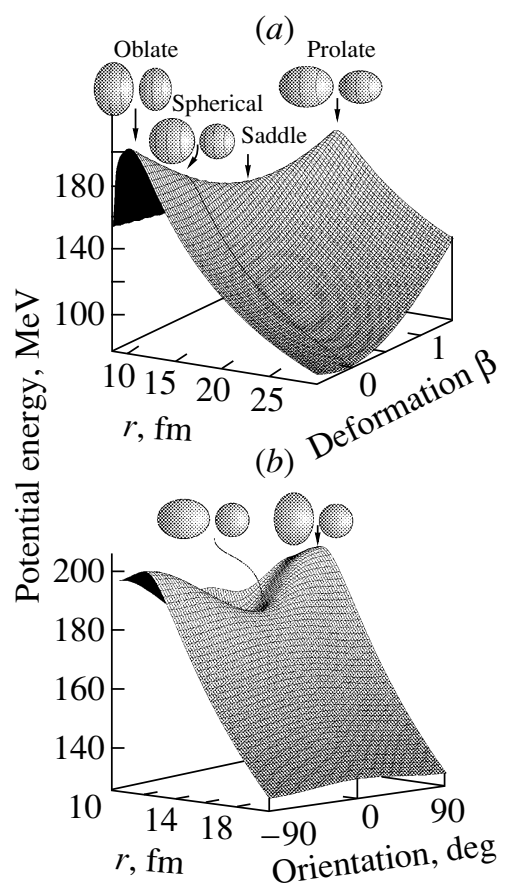

Fig. 1. (a) Potential energy of ${ }^{48} \mathrm{Ca}+{ }^{208} \mathrm{~Pb}$ depending on distance and quadrupole dynamic deformations of both nuclei. ( $b$ ) Potential energy of ${ }^{48} \mathrm{Ca}+{ }^{238} \mathrm{U}$ depending on orientation of statically deformed ${ }^{238} \mathrm{U}$ nucleus $\left(\beta_{2}^{\text {g.s }}=\right.$ $0.215)$.

tion is the most vague. It is due to the fact that, in the fusion of light and medium nuclei, in which the fissility of the $\mathrm{CN}$ is not very high, the colliding nuclei having overcome the Coulomb barrier form a $\mathrm{CN}$ with a probability $P_{\mathrm{CN}} \approx 1$. Thus, this reaction stage does not influence the yield of ER at all. However, in the fusion of heavy nuclei, it is the fission channels (normal and quasifission) that substantially determine the dynamics of the whole process; the $P_{\mathrm{CN}}$ value can be much smaller than unity, while its accurate calculation is very difficult. Setting $P_{x n}=1$ in (1.1), we get the cross section of $\mathrm{CN}$ formation $\sigma_{\mathrm{CN}}$, which can be measured by detection of ERs and fission fragments forming in normal fission (if they are distinguished from quasifission fragments and from products of deep-inelastic collision). Setting in addition $P_{\mathrm{CN}}=1$ in (1.1), we get the capture cross section $\sigma_{\text {cap }}$, which can be measured by detection of all fission fragments (if they are distinguished from products of deep inelastic collision). It is clear that, for symmetric fusion reactions, $\sigma_{\mathrm{CN}}$ and $\sigma_{\text {cap }}$ cannot be measured experimentally.

\section{CAPTURE CROSS SECTION}

The Bass approximation of the potential energy of the interaction between two heavy spherical nuclei [4] is widely used and reproduces rather well

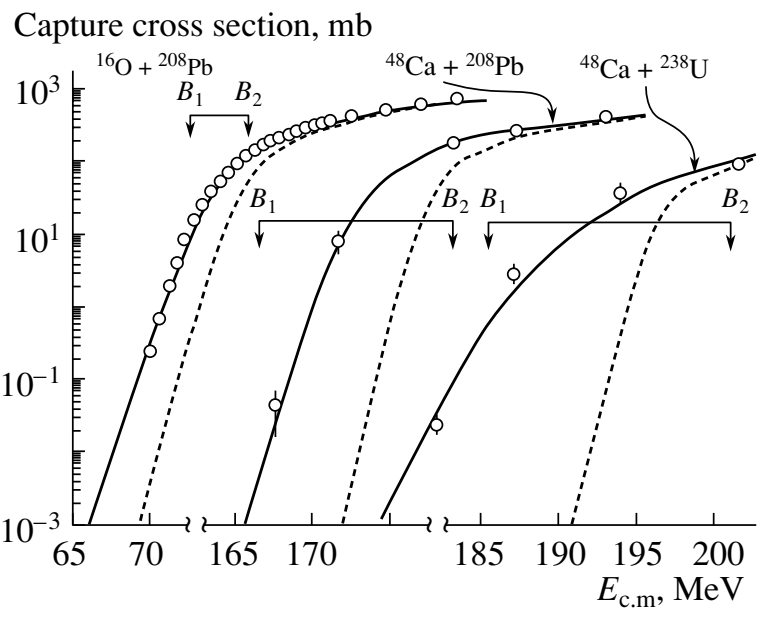

Fig. 2. Capture cross sections in the ${ }^{16} \mathrm{O}+{ }^{208} \mathrm{~Pb}$ [9], ${ }^{48} \mathrm{Ca}+{ }^{208} \mathrm{~Pb}$ [10], and ${ }^{48} \mathrm{Ca}+{ }^{238} \mathrm{U}$ [3] fusion reactions. Dashed curves represent one-dimensional barrier penetration calculations with the Bass barriers. Solid curves show the effect of dynamic deformation of nuclear surfaces (first two reactions) and orientation of statically deformed nuclei $\left({ }^{48} \mathrm{Ca}+{ }^{238} \mathrm{U}\right.$ case $)$. The arrows marked by $B_{1}$ and $B_{2}$ show the positions of the corresponding Coulomb barriers (see the text).

the height of the potential barrier. Coupling with the excitation of nuclear collective states (surface vibrations and/or rotation of deformed nuclei) and with nucleon transfer channels significantly influences the capture cross section at near-barrier energies. In Fig. 1, the potential energy is shown depending on dynamic deformation of spherical nuclei ${ }^{48} \mathrm{Ca}+{ }^{208} \mathrm{~Pb}$ and on mutual orientation of deformed nuclei ${ }^{48} \mathrm{Ca}+{ }^{238} \mathrm{U}\left(\beta_{2}^{\mathrm{g} . \mathrm{s}}=0.215\right)$. The incoming flux has to overcome, in fact, a multidimensional ridge with its height depending on orientation and/or dynamic deformation. This means that we have to talk not about one barrier $B$ but rather about a "barrier distribution."

In $[5,6]$, a semiempirical approach was proposed for calculating the penetration probability of such multidimensional potential barriers. Calculating the barriers $B_{1}$ and $B_{2}$ for two limit configurations [in the case of statically deformed nuclei, they correspond to the tip and side orientations, otherwise they correspond to the so-called saddle dynamic deformation (see Fig. 1a) and spherical configuration], we may approximate the barrier distribution function [7] by an asymmetric Gaussian centered at $B_{0}=\left(B_{1}+B_{2}\right) / 2$. Approximating the radial dependence of the barrier by a parabola and using the Hill-Wheeler formula [8] for the penetration probability of the one-dimensional potential barrier, we may estimate the quantum pen- 
etrability of the multidimensional barrier as follows:

$$
\begin{aligned}
& P_{\text {cont }}(E, l)=\int f(B)\left[1+\exp \left(\frac{2 \pi}{\hbar \omega(l)}\right.\right. \\
& \left.\left.\times\left[B+\frac{\hbar^{2}}{2 \mu R_{B}^{2}(l, B)} l(l+1)-E\right]\right)\right]^{-1} d B .
\end{aligned}
$$

Here, $\hbar \omega_{B}$ is defined by the width of the parabolic barrier, $R_{B}$ defines the position of the barrier, and the barrier distribution function satisfies the normalization condition $\int f(B) d B=1$.

The capture cross sections calculated within this approach are shown in Fig. 2 for the three reactions (solid curves). They are compared with theoretical calculations made within a model of onedimensional barrier penetrability for spherical nuclei (dashed curves). In all three cases, a substantial increase in the barrier penetrability is observed in the subbarrier energy region. However, the character of this increase significantly changes: the shift of the barrier and the distribution width, in particular, grow with the increase in the masses of fusing nuclei. An additional decrease in the ${ }^{48} \mathrm{Ca}+{ }^{238} \mathrm{U}$ capture cross section at above-barrier energies as compared with its geometrical limit is explained by a much shallower potential pocket and, thus, by a much smaller value of the critical angular momentum.

Good agreement between the calculated and experimental capture cross sections allows us to believe that we may get a rather reliable estimation of the capture cross section for a given projectiletarget combination if there are no experimental data or if these data cannot be obtained at all (symmetric combinations). However, we should realize that some uncertainty nevertheless remains in choosing the parameters defining the multidimensional potential barrier and the capture cross section [6]. The role of the neutron exchange is also not clear yet. Thus, in the cases of fusion of very heavy nuclei and especially for symmetric fusion reactions, the accuracy of our current predictions of the capture cross sections in the subbarrier energy region is about one order of magnitude. At above-barrier energies, this accuracy is much better.

\section{FUSION-FISSION DYNAMICS}

The processes of the $\mathrm{CN}$ formation and quasifission are the least studied stages of the heavy-ion fusion reaction. To solve this problem, we have to answer very fundamental questions. What are the main degrees of freedom playing most important role at this reaction stage? What is the corresponding driving potential and what is an appropriate equation of motion for description of time evolution of the nuclear system at this stage? Today, there is no
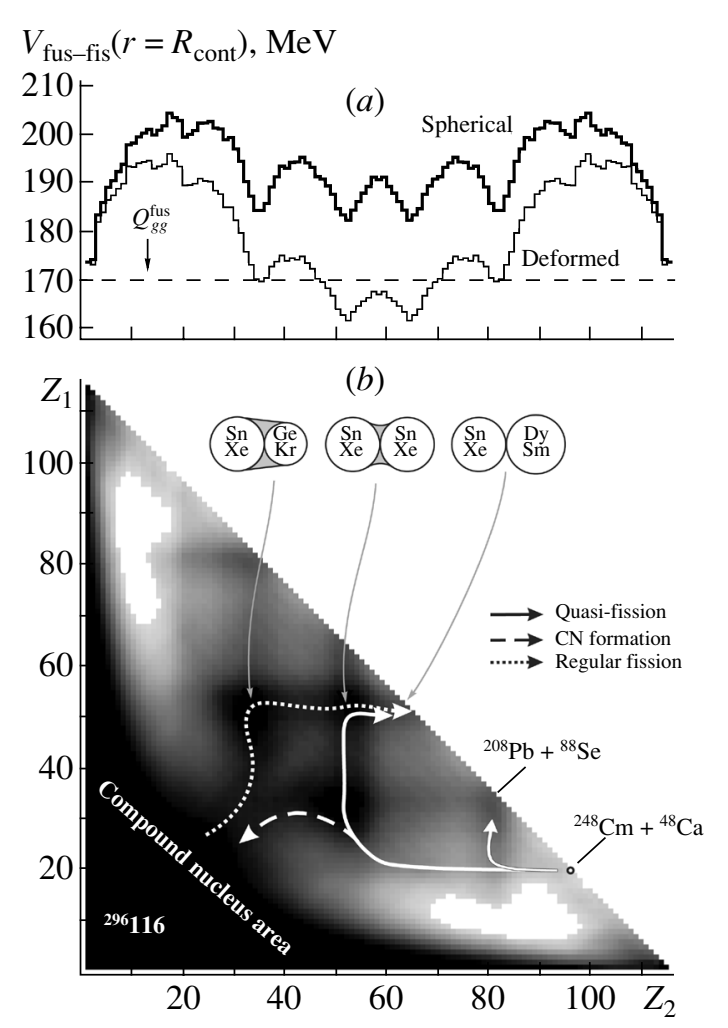

Fig. 3. Driving potential $V_{\text {fus-fis }}\left(Z_{1}, Z_{2}\right)$ of the nuclear system consisting of 116 protons and 180 neutrons. (a) Potential energy of two touching nuclei at $A_{1}+A_{2}=$ $A_{\mathrm{CN}}, \Delta A=0$, i.e., along the diagonal of the lower figure. The thick line corresponds to the case of spherical nuclei, whereas the thin line corresponds to $\delta_{1}+\delta_{2}=0.3$. ( $b$ ) Topographical landscape of the driving potential on the plane $\left(Z_{1}, Z_{2}\right)$ (zero deformations). The dark regions correspond to the lower potential energies (more compact configurations).

consensus for the answers and for the mechanism of the compound nucleus formation itself, and quite different, sometimes opposite in their physics sense, models are used for its description.

In $[5,11]$, a new approach was proposed for description of fusion-fission dynamics based on a simplified semiempirical version of the two-center shell model idea [12]. It is assumed that, on a path from the initial configuration of two touching nuclei to the $\mathrm{CN}$ configuration and on a reverse path to the fission channels, the nuclear system consists of two cores $\left(Z_{1}, N_{1}\right)$ and $\left(Z_{2}, N_{2}\right)$ surrounded by a certain number of common (shared) nucleons, $\Delta A=$ $A_{\mathrm{CN}}-A_{1}-A_{2}$, moving in the whole volume occupied by the two cores. The processes of $\mathrm{CN}$ formation, fission, and quasifission take place in the space $\left(Z_{1}, N_{1}, \delta_{1} ; Z_{2}, N_{2}, \delta_{2}\right)$, where $\delta_{1}$ and $\delta_{2}$ are the dynamic deformations of the cores. The compound nucleus is finally formed when two fragments $A_{1}$ and 
(a)

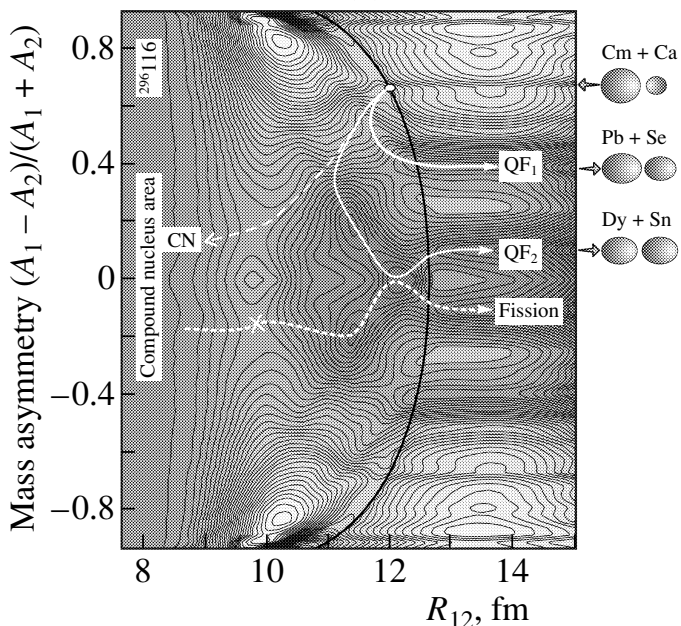

(b)

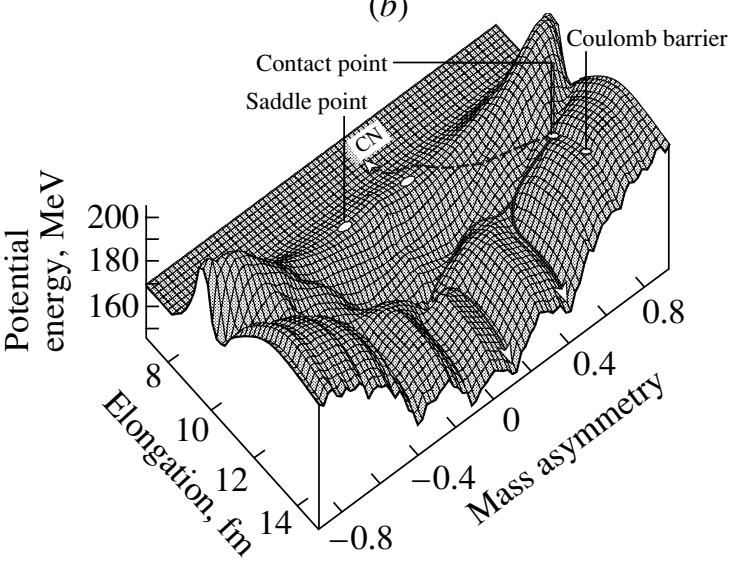

Fig. 4. Driving potential $V_{\text {fus-fis }}$ as a function of mass asymmetry and distance between centers of two nuclei with the deformations $\delta_{1}+\delta_{2}=0.3$, topographical landscape $(a)$ and three-dimensional plot $(b)$. The black solid curve in $(a)$ shows the contact configurations. The paths $\mathrm{QF}_{1}$ and $\mathrm{QF}_{2}$ lead to the asymmetric and nearsymmetric quasifission channels, the dashed curve shows the most probable way to formation of the compound nucleus, and the dotted curve corresponds to normal (regular) fission. See the conformity with Fig. 3.

$A_{2}$ go into its volume, i.e., at $R\left(A_{1}\right)+R\left(A_{2}\right)=R_{\mathrm{CN}}$ or at $A_{1}^{1 / 3}+A_{2}^{1 / 3}=A_{\mathrm{CN}}^{1 / 3}$.

The corresponding driving potential $V_{\text {fus-fis }}\left(r, Z_{1}\right.$, $\left.N_{1}, \delta_{1} ; Z_{2}, N_{2}, \delta_{2}\right)$ was derived in [5] and is shown in Fig. 3 as a function of $Z_{1}, Z_{2}$ (minimized over $N_{1}, N_{2}$ and at fixed values of $\delta_{1}+\delta_{2}$ ). It was found that the microscopic two-center shell model calculations give very close values of potential energy, though slightly less structural. There are several advantages of the proposed approach. The driving potential is derived on the basis of experimental binding energies of two cores, which means that the "true" shell structure is taken into account (see Fig. 3). The driving potential is defined in the whole region $R_{\mathrm{CN}}<r<\infty$, (a)

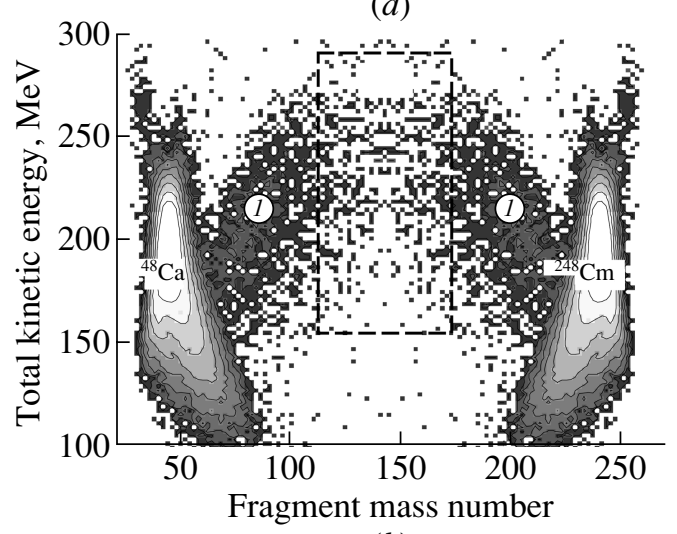

(b)

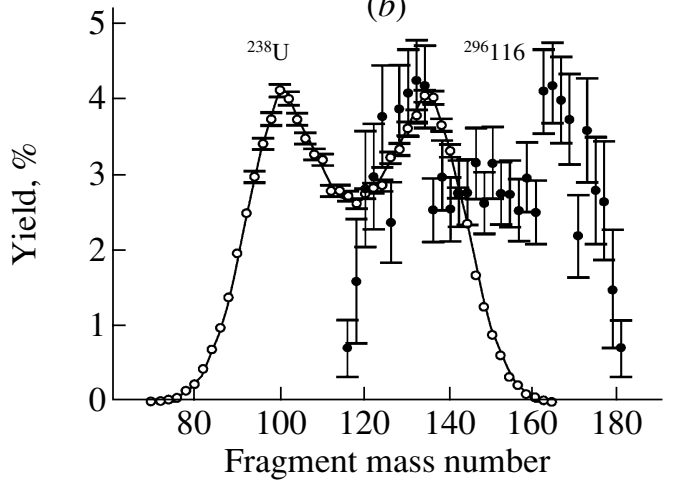

Fig. 5. (a) Two-dimensional TKE-mass plot. The asymmetric quasifission process $\left(\mathrm{QF}_{1}\right.$ path in Fig. 4) contributes mainly to the regions marked by 1 . Normal fission and near-symmetric quasifission $\left(\mathrm{QF}_{2}\right.$ path in Fig. 4) contribute to the region marked by dashed quadrangle. (b) Mass distribution of near-symmetric fission fragments [dashed quadrangle on panel $(a)$ ] detected in the ${ }^{48} \mathrm{Ca}+{ }^{248} \mathrm{Cm}$ reaction at excitation energy of $E^{*}=$ $33 \mathrm{MeV}$ compared with the fission of ${ }^{238} \mathrm{U}$ measured at approximately the same excitation energy [13].

it is a continuous function at $r=R_{\text {cont }}$, and it gives a realistic Coulomb barrier at $r=R_{B}>R_{\text {cont }}$. At last, instead of using the variables $\left(Z_{1}, N_{1} ; Z_{2}, N_{2}\right)$, we may easily recalculate the driving potential as a function of mass asymmetry $\left(A_{1}-A_{2}\right) /\left(A_{1}+A_{2}\right)$ and elongation $R_{12}=r_{0}\left(A_{1}^{1 / 3}+A_{2}^{1 / 3}\right)$ (at $r \geq R_{\text {cont }}$, $R_{12}=r=s+R_{1}+R_{2}$, where $s$ is the distance between nuclear surfaces). These variables along with deformation $\delta_{1}+\delta_{2}$ are commonly used for description of the fission process. The corresponding driving potential is shown in Fig. 4.

As can be seen from Figs. 3 and 4, the shell structure, clearly revealing itself in the contact of two nuclei (Fig. $3 a$ ), is also retained at $\Delta A \neq 0$ ( $R_{12}<R_{\text {cont }}$ ) (see the deep minima in the regions of $Z_{1,2} \sim 50$ and $Z_{1,2} \sim 82$ in Fig. $3 b$ ). Following the fission path (dotted curves in Figs. $3 b$ and $4 a$ ), the system overcomes a multihumped fission barrier, which is well known in fission dynamics. The 


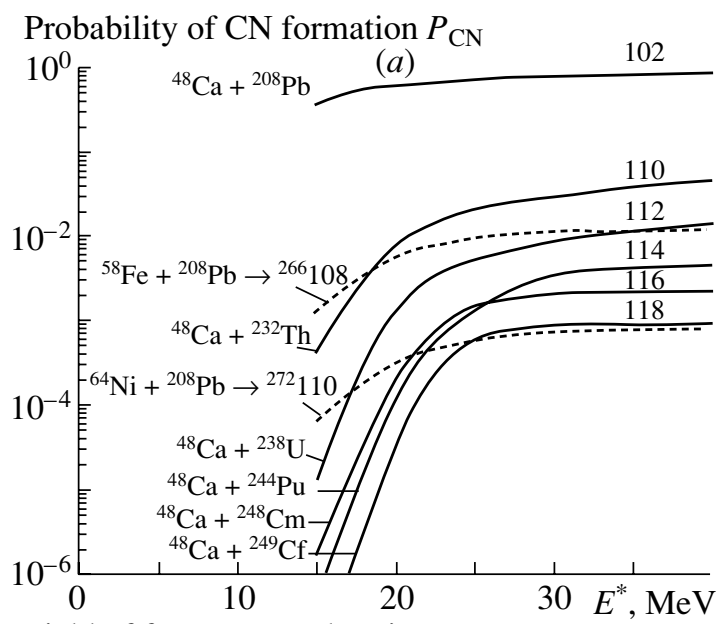

Yield of fragments, rel. units

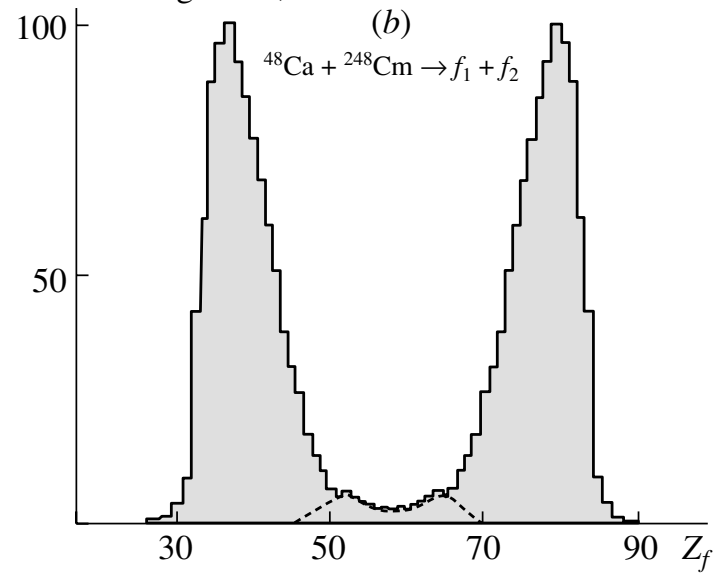

Fig. 6. (a) Probability of compound-nucleus formation for the "hot" ( solid curves) and "cold" (dashed curves) fusion reactions. (b) Charge distribution of quasifission fragments in the ${ }^{48} \mathrm{Ca}+{ }^{248} \mathrm{Cm}$ fusion reaction at $E^{*}=$ $40 \mathrm{MeV}$ (linear scale, relative units). The main peaks correspond to the path $\mathrm{QF}_{1}$ in Fig. 4 (see region 1 in Fig. $5 a$ ), whereas the small near-symmetric peaks correspond to the path $\mathrm{QF}_{2}$.

intermediate minima correspond to the shape isomer states. From our analysis, we may definitely conclude that these isomeric states are nothing else but twocluster configurations with magic or semimagic cores (see Fig. 3b).

As regards the superheavy compound nucleus formation in the fusion reaction ${ }^{48} \mathrm{Ca}+{ }^{248} \mathrm{Cm}$, one can see that, after the contact, the nuclear system may easily decay into the quasifission channels (mainly asymmetric: $\mathrm{Se}+\mathrm{Pb}, \mathrm{Kr}+\mathrm{Hg}$; also near-symmetric: $\mathrm{Sn}+\mathrm{Dy}, \mathrm{Te}+\mathrm{Gd})$ - solid arrow lines in Figs. $3 b$ and 4. Only a small part of the incoming flux reaches a CN configuration (dashed-arrow line). An experimental two-dimensional total kinetic energy (TKE) mass plot for the ${ }^{48} \mathrm{Ca}+{ }^{248} \mathrm{Cm}$ fusion-fission reaction [3] is shown in Fig. 5. The experimental data are quite

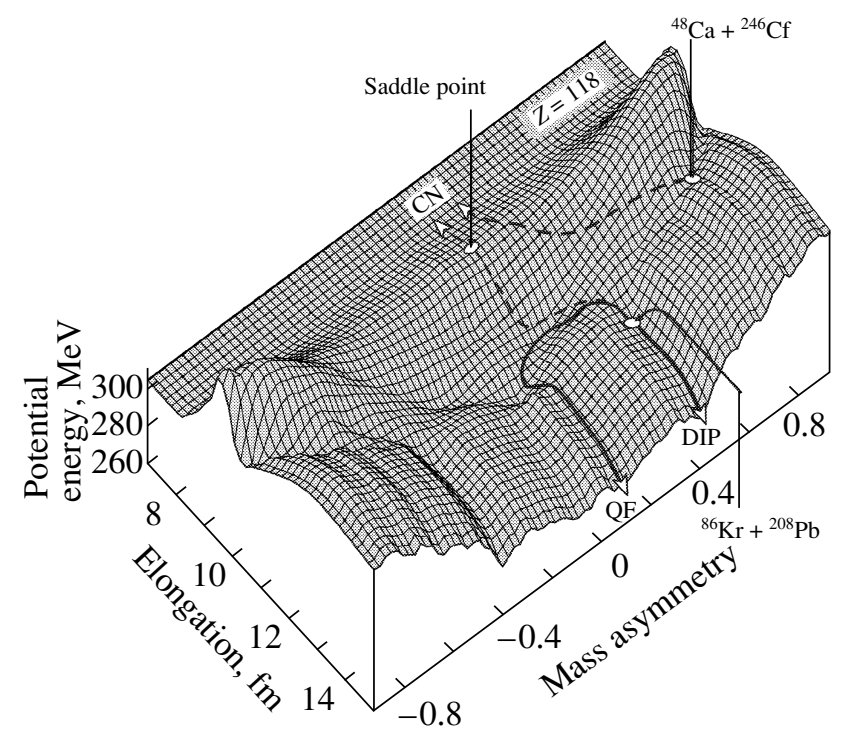

Fig. 7. Fusion-fission driving potential for the nucleus ${ }^{294} 118$ formed in the ${ }^{86} \mathrm{Kr}+{ }^{208} \mathrm{~Pb}$ and ${ }^{48} \mathrm{Ca}+{ }^{246} \mathrm{Cf}$ fusion reactions.

understandable qualitatively in terms of multidimensional potential energy surface shown in Figs. 3 and 4.

Using the driving potential $V_{\text {fus-fis }}\left(Z_{1}, N_{1}, Z_{2}\right.$, $N_{2}, \delta_{1}, \delta_{2}$ ), we may determine the probability of $\mathrm{CN}$ formation $P_{\mathrm{CN}}\left(A_{1}+A_{2} \rightarrow C\right)$, being part of expression (1.1) for the cross section of the synthesis of superheavy nuclei. It can be done, for example, by solving the master equation [14] for the distribution function $F\left(Z_{1}, N_{1}, Z_{2}, N_{2}, \delta_{1}, \delta_{2} ; t\right)$. The probability of $\mathrm{CN}$ formation is determined as an integral of the distribution function over the region $R_{1}+R_{2} \leq R_{\mathrm{CN}}$. Similarly, one can define the probabilities of finding the system in different channels of quasifission, i.e., the charge and mass distribution of fission fragments measured experimentally.

Results of such calculations performed with a restricted number of variables are shown in Fig. 6. For the "hot" fusion reactions, based on using ${ }^{48} \mathrm{Ca}$ as a projectile, the probability of $\mathrm{CN}$ formation at first falls very sharply with increasing $Z_{\mathrm{CN}}$, but then it remains at the level of $10^{-3}$ for $Z_{\mathrm{CN}}=114-118$ at excitation energies $E^{*} \geq 30 \mathrm{MeV}$. Such behavior of $P_{\mathrm{CN}}$ reflects the fact of insignificant changes of $V_{\text {fus-fis }}$ for all these reactions. In contrast with that, for the "cold" fusion reactions, based on using ${ }^{208} \mathrm{~Pb}$ as a target, the probability of $\mathrm{CN}$ formation decreases very fast with increasing $Z_{\mathrm{CN}}$ (see dashed curves in Fig. $6 a$ ). A qualitative explanation of that can be made again in terms of potential energy surface. In Fig. 7, the driving potential is shown for the synthesis of nucleus ${ }^{294} 118$ in the "cold" fusion reaction ${ }^{86} \mathrm{Kr}+{ }^{208} \mathrm{~Pb}$. Due 
Cross section, mb
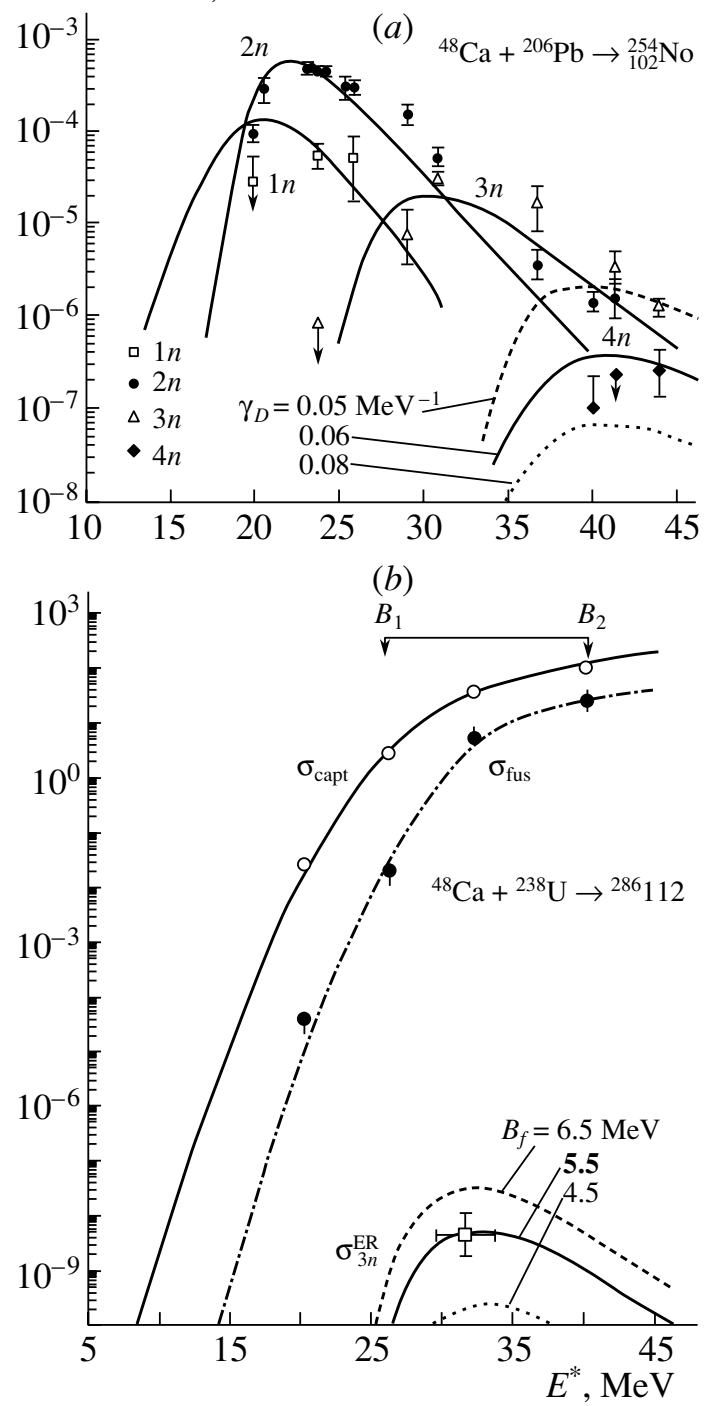

Fig. 8. (a) Cross sections of the evaporation residue production for different $x n$ channels in the ${ }^{48} \mathrm{Ca}+{ }^{206} \mathrm{~Pb}$ fusion reaction. The experimental data are from [17]. The solid curves correspond to the calculations with the damping factor $\gamma_{D}=0.06 \mathrm{MeV}^{-1}$, whereas the dashed and dotted curves for the $4 n$ channel are calculated with $\gamma_{D}=0.05$ and $0.08 \mathrm{MeV}^{-1}$, respectively. $(b)$ The capture cross section (all fission fragments, open circles), the total yield of near-symmetric fission fragments with $A=$ $A_{\mathrm{CN}} / 2 \pm 20$ (solid circles), and the evaporation residue production cross section in the $3 n$ channel of the ${ }^{48} \mathrm{Ca}+$ ${ }^{238} \mathrm{U}$ reaction. The arrows show the Coulomb barriers for two ultimate orientations of the deformed target nucleus. The cross section of evaporation residue formation was calculated with a fission barrier of 4.5 (dotted curve), 5.5 (solid curve), and 6.5 MeV (dashed curve).

to dynamic deformation of both nuclei, the potential energy at the point of contact is even lower than the energy of the $\mathrm{CN}$ ground state. The nuclear system has to evolve upward on the potential energy surface to reach the $\mathrm{CN}$ configuration. It is possible only at sufficiently high excitation energy. Disappearance of the locked Coulomb barrier makes the system unstable against reseparation into the deep-inelastic and quasielastic channels, which have to dominate in this reaction at low energies. In contrast with that, in more asymmetric case of ${ }^{48} \mathrm{Ca}+{ }^{246} \mathrm{Cf}$ fusion reaction leading to the same $\mathrm{CN}$, the potential energy at the point of contact is above the ground state of the $\mathrm{CN}$, and the nuclear system evolves down along the potential energy surface. Of course, the main flux goes to the quasifission valley $(\mathrm{Kr}+\mathrm{Pb})$, but nevertheless a small part of it reaches the $\mathrm{CN}$ configuration (dashed line in Fig. 7).

Exploration of the multidimensional fusion-fission driving potential itself and of the corresponding evolution of a heavy-nuclear system along its surface is a very promising and fruitful experimental problem. For that purpose, one may perform, for example, fusion-fission and ER measurements on formation of the same easily fissile ${ }^{224} \mathrm{U}$ nucleus in different projectile-target combinations: ${ }^{20} \mathrm{Ne}+{ }^{204} \mathrm{~Pb}$ (very asymmetric, behind the Businaro-Gallone barrier), ${ }^{64} \mathrm{Ni}+{ }^{160} \mathrm{Gd}$ (less asymmetric, in front of the Businaro-Gallone barrier), ${ }^{88} \mathrm{Sr}+{ }^{136} \mathrm{Xe}$ and ${ }^{100} \mathrm{Mo}+{ }^{124} \mathrm{Sn}$ (symmetric, inverse to fission process), ${ }^{86} \mathrm{Kr}+{ }^{138} \mathrm{Ba}$ (closed shell nuclei, suppressed deep-inelastic channels), and ${ }^{76} \mathrm{Ge}+{ }^{148} \mathrm{Nd}$ (deformed, dependence on orientation).

\section{FISSION BARRIERS OF SUPERHEAVY NUCLEI}

The survival probability $P_{x n}\left(l, E^{*}\right)$ of a cooling excited compound nucleus can be calculated within a statistical model $[6,15]$. The most uncertain parameter here is the fission barrier. For nuclei with $Z>100$, which cannot be used as a target material, experimental measurement of the fission barriers is not possible. Calculating the fission barrier for the atomic nucleus (mainly its microscopic component) is also a very complicated puzzle faced with the necessity of solving a many-body quantum problem. The exact solution to that problem is currently unobtainable, and the accuracy of the approximations in use is rather difficult to estimate. As a result, the fission barriers for superheavy nuclei calculated within the different approaches differ greatly (by several megaelectronvolts). Any experimental information on the fission barriers of those nuclei seems to be highly valuable.

An important property of the fission barrier is that it has a pronounced effect on the survival probability of an excited nucleus in its cooling by emitting neutrons and $\gamma$ rays in competition with fission. It 
is this property that may be taken advantage of to make an estimate of the fission barrier of a superheavy nucleus if it is impossible to measure the fission barrier directly. Higher sensitivity may be obtained if such competition is tested several times during the evaporation cascade ("hot" fusion reactions). In this case, the cross section $\sigma_{\mathrm{ER}}^{x n}(E)$, which is proportional roughly to $\left(\Gamma_{n} / \Gamma_{f}\right)^{x}$, happens to be more sensitive to the value of the fission barrier since it increases in importance by a factor of $x$. For the experimental value of the survival probability of the superheavy nucleus to be deduced, it is necessary to measure the cross section of weakly excited $\mathrm{CN}$ production in the near-barrier fusion of heavy ions as well as the cross section for the yield of a heavy evaporation residue. It was experiments of this kind that were carried out at FLNR (JINR, Dubna) recently [1-3] as part of a series of experiments on the production of nuclei with $Z=112,114$, and 116 formed in the $3 n$ and $4 n$ evaporation channels.

The fission barriers are usually calculated according to the formula $B_{f}(J=0)=B_{\mathrm{LD}}-\delta W e^{-\gamma_{D} E^{*}}$, where $B_{\mathrm{LD}}$ is the liquid-drop fission barrier [16], which is negligibly small for nuclei with $Z>112$; $\delta W$ is the shell correction for the ground-state energy; and $\gamma_{D}$ is the damping parameter, which accounts for the fact that shell effects fall off as the excitation energy of the $\mathrm{CN}$ increases. The value of this parameter is especially important in the case of superheavy nuclei, whose fission barriers are mainly determined just by the shell corrections for their ground states. In the literature, one can find close but slightly different values for the damping parameter, and we paid special attention to the sensitivity of the calculated cross sections to this parameter. Figure $8 a$ shows how much the cross section for the $4 n$ channel is sensitive to a change in the damping parameter. Simultaneous analysis of a great number of "hot" fusion reactions used for producing heavy elements allows the conclusion that the value of this parameter lies in the range $\gamma_{D}^{-1}=14-18 \mathrm{MeV}$.

After calculating the value of $P_{\text {cont }}(E, l)$ in such a way as for the measured capture cross section to be reproduced and parametrizing the $\mathrm{CN}$ production probability $P_{\mathrm{CN}}$ in such a way as for $\sigma_{\mathrm{CN}}^{\exp }$ to be reproduced, fission barriers for the nuclei of the evaporation cascade can be chosen in such a way as for the corresponding measured cross section of the yield of a heavy evaporation residue nucleus to be reproduced with the help of (1.1). The calculated results are shown in Fig. 8 for the case of the ${ }^{48} \mathrm{Ca}+{ }^{238} \mathrm{U} \rightarrow{ }^{286} 112$ fusion reaction. Taking account of the fact that fission barriers vary not so much from nucleus to nucleus in an evaporation cascade, as well as making the procedure for assessing them
The lower limits of the heights of fission barriers

\begin{tabular}{c|c|c|c|c|c}
\hline Nucleus & $\begin{array}{c}E^{*}, \\
\mathrm{MeV}\end{array}$ & $\begin{array}{c}\sigma_{\text {capt }}, \\
\mathrm{mb}\end{array}$ & $\begin{array}{c}\sigma_{\text {fus }}, \\
\mathrm{mb}\end{array}$ & $\begin{array}{c}\sigma_{\mathrm{ER}}, \\
\mathrm{pb}\end{array}$ & $\begin{array}{c}\left\langle B_{f}\right\rangle, \\
\mathrm{MeV}\end{array}$ \\
\hline 286112 & 31.5 & 40 & $\leq 5$ & $5.0_{-3.2}^{+6.3}(3 n)$ & $\geq 5.5$ \\
292114 & 36.5 & 30 & $\leq 4$ & $0.5_{-0.3}^{+0.8}(4 n)$ & $\geq 6.7$ \\
296116 & 34.8 & 30 & $\leq 2$ & $0.5_{-0.3}^{+0.8}(4 n)$ & $\geq 6.4$ \\
\hline
\end{tabular}

simpler, the same value $B_{f}$ was used for these nuclei. The typical sensitivity of the calculated production cross section for the ER to a change in the value of the fission barrier is shown in Fig. 8. It is the fact that this sensitivity is high which allows one to expect the value of the fission barrier to be deduced to an accuracy of the order of $\pm 0.5 \mathrm{MeV}$ with allowance made for the experimental error in measuring this cross section and the uncertainty of some parameters used in the calculations [6]. Since, as discussed above in Section 3, the production probability for a true compound nucleus may really be less than the value of $\sigma_{\mathrm{CN}}^{\exp } / \sigma_{\text {capt }}^{\exp }$, then comparing the measured and calculated cross sections for the evaporation residues allows one to deduce in fact the lower limits for the fission barriers of the corresponding nuclei. Final results are presented in the table.

The analysis of the available experimental data on the fusion and fission of the nuclei of ${ }^{286} 112,{ }^{292} 114$, and ${ }^{296} 116$ produced in the reactions ${ }^{48} \mathrm{Ca}+{ }^{238} \mathrm{U}$, ${ }^{48} \mathrm{Ca}+{ }^{244} \mathrm{Pu}$, and ${ }^{48} \mathrm{Ca}+{ }^{248} \mathrm{Cm}$ [3], as well as experimental data on the survival probability of those nuclei in evaporation channels of three- and fourneutron emission [1,2], enables the quite reliable conclusion that the fission barriers of those nuclei are really quite high, which results in their relatively high stability. The lower limits that we have obtained for the fission barriers of nuclei of ${ }^{283-286} 112,{ }^{288-292} 114$, and ${ }^{292-296} 116$ are $5.5,6.7$, and $6.4 \mathrm{MeV}$, respectively [18].

\section{CROSS SECTIONS OF SUPERHEAVY-ELEMENT PRODUCTION}

Calculating the capture cross sections and the probability for $\mathrm{CN}$ formation as described above and using the fission barriers based on the ground-state shell corrections of Möller et al. [19], we estimated the cross sections of superheavy element formation in the "hot" and "cold" fusion reactions leading to heavy nuclei with $Z_{\mathrm{CN}} \geq 102$ (Fig. 9). The cross sections for formation of superheavy nuclei with $Z=114-118$ in the $3 n$ and $4 n$ evaporation channels of the "hot" 


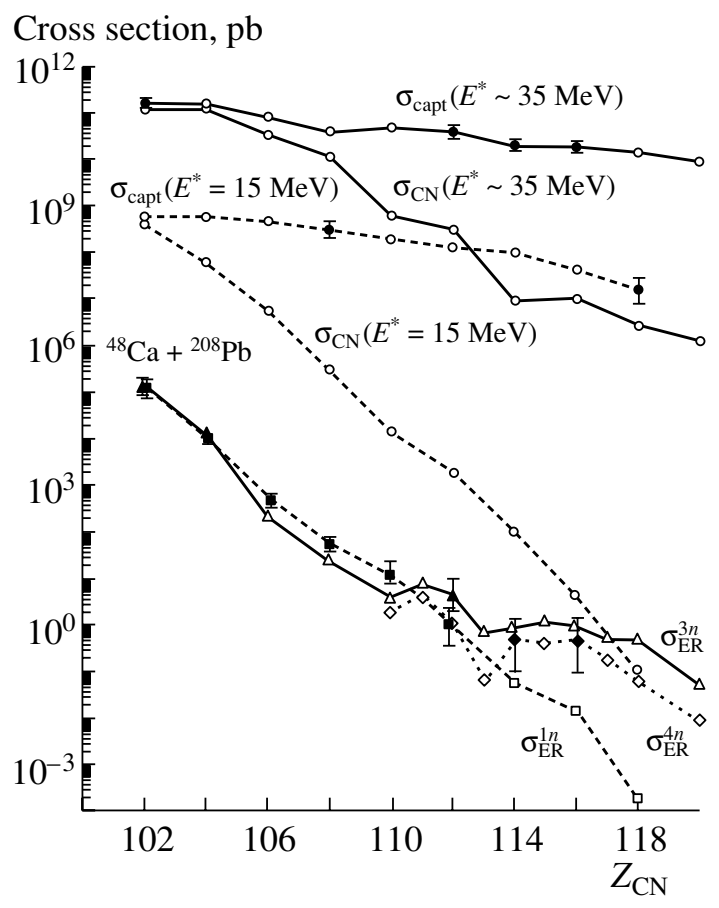

Fig. 9. Capture, fusion, and evaporation residue formation cross sections. For "hot" fusion reactions, the triangles show maximal values of $\sigma_{\mathrm{ER}}$ in the $3 n$ evaporation channel, and the diamonds correspond to the $4 n$ evaporation channel. Evaporation residue cross sections for the "cold" fusion reactions ( $1 n$ evaporation channel) are shown by squares. The closed symbols correspond to the experimental values, whereas the open ones correspond to the calculated cross sections. For the "hot" fusion reactions the following projectile-target combinations are used: ${ }^{48} \mathrm{Ca}+{ }^{208} \mathrm{~Pb},{ }^{12} \mathrm{C}+{ }^{249} \mathrm{Cf},{ }^{18} \mathrm{O}+{ }^{249} \mathrm{Cf}$, ${ }^{26} \mathrm{Mg}+{ }^{248} \mathrm{Cm}$; for $110 \leq Z_{\mathrm{CN}} \leq 118,{ }^{48} \mathrm{Ca}$ is used as a projectile and ${ }^{232} \mathrm{Th},{ }^{231} \mathrm{~Pa},{ }^{238} \mathrm{U},{ }^{237} \mathrm{~Np},{ }^{244} \mathrm{Pu},{ }^{243} \mathrm{Am}$, ${ }^{248} \mathrm{Cm},{ }^{247} \mathrm{Bk}$, and ${ }^{249} \mathrm{Cf}$ are the targets. The last combination leading to $Z_{\mathrm{CN}}=120$ is ${ }^{58} \mathrm{Fe}+{ }^{244} \mathrm{Pu}$. For the "cold" fusion reactions, ${ }^{208} \mathrm{~Pb}$ is used as a target and the projectiles are the heaviest isotopes of the corresponding stable nuclei (from ${ }^{48} \mathrm{Ca}$ to ${ }^{86} \mathrm{Kr}$ ).

fusion reactions were found to be at the level of $0.1-$ $1.0 \mathrm{pb}$. For the available experimentally "cold" fusion reactions, the cross sections for formation of the same elements in the $1 n$ evaporation channel are much lower. A gain of about three orders of magnitude in the survival probability, $P_{1 n}\left(E^{*} \approx 15 \mathrm{MeV}\right) / P_{3 n}\left(E^{*} \approx\right.$ $35 \mathrm{MeV}) \approx 10^{3}$, is compensated here by a loss of two orders of magnitude in the capture cross sections and more than two orders of magnitude in the probability of $\mathrm{CN}$ formation.

\section{CONCLUSION}

From the analysis of the multidimensional fusionfission driving potential, we may conclude that, in the fission process, a weakly excited heavy nucleus on its way from the ground state to the scission point passes through the optimal configurations with minimal potential energies (shape isomer states), which are nothing else but the two-cluster configurations with magic (closed shell) cores. Analysis of the experimental data on the fusion and fission of the nuclei of ${ }^{286} 112,{ }^{292} 114$, and ${ }^{296} 116$, as well as experimental data on the survival probability of those nuclei in evaporation channels of three- and four-neutron emission, enables us to make the quite reliable conclusion that the fission barriers of those nuclei are really quite high, which results in their relatively high stability. The lower limits that we have obtained for the fission barrier heights of ${ }^{283-286} 112,{ }^{288-292} 114$, and ${ }^{292-296} 116$ nuclei are 5.5, 6.7, and $6.4 \mathrm{MeV}$, respectively. This makes the synthesis of superheavy nuclei with $112 \leq Z \leq 120$ in asymmetric fusion reactions experimentally attainable (see Fig. 9). The choice of appropriate projectile-target combination is very important here. For example, using the fission barriers predicted in [19], we found that the ER cross section for production of element 116 in the $3 n$ evaporation channel of the ${ }^{48} \mathrm{Ca}+{ }^{247} \mathrm{Cm}$ fusion reaction should be about $1.5 \mathrm{pb}$ at $35 \mathrm{MeV}$ of initial excitation energy of the $\mathrm{CN}$. That is due to more favorable evaporation of two odd neutrons with lower separation energies compared with a synthesis of the same element in the ${ }^{48} \mathrm{Ca}+{ }^{248} \mathrm{Cm}$ reaction.

\section{ACKNOWLEDGMENTS}

The work was supported by INTAS, grant no. 00655.

\section{REFERENCES}

1. Yu.Ts. Oganessian, A. V. Yeremin, A. G. Popeko, et al., Nature 400, 242 (1999).

2. Yu. Ts. Oganessian, V. K. Utyonkov, Yu. V. Lobanov, et al., Yad. Fiz. 63, 1769 (2000) [Phys. At. Nucl. 63, 1679 (2000)]

3. M. G. Itkis, Yu. Ts. Oganessian, A. A. Bogatchev, et al., in Proceedings of the International Workshop on Fusion Dynamics at the Extremes, Dubna, 2000 , Ed. by Yu. Ts. Oganessian and V. I. Zagrebaev (World Sci., Singapore, 2001), p. 93.

4. R. Bass, Nuclear Reactions with Heavy Ions (Springer-Verlag, Berlin, 1980), p. 326.

5. V. I. Zagrebaev, Phys.Rev. C 64, 034606 (2001).

6. V. I. Zagrebaev, Y. Aritomo, M. G. Itkis, et al., Phys. Rev. C 65, 014607 (2002).

7. N. Rowley, G. R. Satchler, and P. H. Stelson, Phys. Lett. B 254, 25 (1991).

8. D. L. Hill and J. A. Wheeler, Phys. Rev. 89, 1102 (1953).

9. C. R. Morton, D. J. Hinde, J. R. Leigh, et al., Phys. Rev. C 52, 243 (1995). 
10. M. G. Itkis, Yu. Ts. Oganessian, E. M. Kozulin, et al., Nuovo Cimento A 111,783 (1998).

11. V. I. Zagrebaev, J. Nucl. Radiochem. Sci. 3, 13 (2002)

12. U. Mosel, J. Maruhn, and W. Greiner, Phys. Lett. B 34B, 587 (1971); J. Maruhn and W. Greiner, Z. Phys. 251, 431 (1972).

13. A. Goverdovski, private communication.

14. L. G. Moretto and J. S. Sventek, Phys. Lett. B 58B, 26 (1975).
15. A. V. Ignatyuk, Statistical Properties of Excited Atomic Nuclei (Energoatomizdat, Moscow, 1983).

16. W. D. Myers and W. J. Swiatecki, Ark. Fys. 36, 343 (1967).

17. Yu.Ts. Oganessian, V. K. Utyonkov, Yu. V. Lobanov, et al., Phys. Rev. C 64, 054606 (2001).

18. M. G. Itkis, Yu. Ts. Oganessian, and V. I. Zagrebaev, Phys. Rev. C 65, 044602 (2002).

19. P. Möller, J. R. Nix, W. D. Myers, and W. J. Swiatecki, At. Data Nucl. Data Tables 59, 185 (1995). 